УДК 697.12

\title{
ЧИСЛЕННОЕ МОДЕЛИРОВАНИЕ ТЕПЛОПЕРЕНОСА ЧЕРЕЗ НЕУТЕПЛЕННЫЙ ФУНДАМЕНТ ЗДАНИЯ
}

Басок Б.И., член-корреспондент НАН Украины, Давыденко Б.В., докт. техн. наук, Новиков В.Г., канд. техн. наук, Гончарук С.М., канд. техн. наук, Лысенко О.М.

ІИнститут технической теплофизики НАН Украины, ул. Желябова, 2а, Киев-57, 03057, Украина

Наведено результати чисельного моделювання переносу теплоти 3 будівлі через фундамент у зовнішній простір. Результати порівнюються 3 даними, отриманими за відомими методиками та 3 експериментом. Відзначено вплив геометричної форми фундаменту на рівні тепловтрат.
Представлены результаты численного моделирования переноса теплоты из здания через фундамент во внешнее пространство. Результаты сравниваются с данными, полученными по известным методикам и с экспериментом. Отмечено влияние геометрической формы фундамента на уровни теплопотерь.
The results of numerical modeling of heat transfer through the building foundation to the environment are presented. The results were compared with those obtained by known methods and with experiment. The influence of the geometric shape of the foundation on the levels of heat loss is noticed.

Библ. 3, рис. 4.

Ключевые слова: фундамент здания, тепловые потери, численное моделирование.

$F$, м $^{2}$ - площадь;

$H$, м - глубина грунтового массива;

$Q$, Вт - тепловой поток;

$q, \mathrm{BT} / \mathrm{M}^{2}-$ плотность теплового потока;

$R, \mathrm{M}^{2} \cdot \mathrm{K} / \mathrm{BT}$ - термическое сопротивление;

$\mathrm{t},{ }^{0} \mathrm{C}$ - температура;

x; y; z, м - прямоугольные координаты;

$\alpha, \mathrm{BT} /\left(\mathrm{M}^{2} \cdot \mathrm{K}\right)$ - коэффициент теплоотдачи;

$\Delta, \mathrm{M}-$ толщина;

$\lambda, \mathrm{BT} /(\mathrm{м} \cdot \mathrm{K})$ - коэффициент теплопроводности.

\section{Введение}

Потери теплоты через фундамент здания считаются сравнительно невысокими по сравнению с теплопотерями через другие части ограждающих конструкций. Вместе с тем, точный расчет указанных теплопотерь вследствие сложности процесса теплопереноса из отапливаемого помещения к наружному воздуху через грунт сопряжен с определенными трудностями. Для их учета обычно используется приближенный метод, который предполагает разделение пола нижнего этажа на четыре характерные зоны. Зонами считаются полосы, параллельные боковым стенам шириной 2 м. Нумерация зон производится в зависимости от их расположения относительно боковых стен. Полоса, ближайшая к наружной стене, считается

\section{Нижние индексы:}

max - максимальный;

в - внутренний;

г- грунт;

зд - здание;

н - наружный;

ст - стена;

ф- фундамент.

первой зоной, следующие две полосы - второй и третьей. Остальная поверхность пола считается четвертой зоной. Каждой зоне ставится в соответствие условное сопротивление теплопередаче. Если фундамент здания - неутепленный, и теплопроводность определенных слоев пола и фундамента не ниже 1,2 Вт/(м·К), значения условных сопротивлений теплопередаче принимаются равными: $\mathrm{R}_{1}=2,1$ м $^{2} \mathrm{~K} / \mathrm{B}$ - для первой зоны; $\mathrm{R}_{2}=4,3$ $\mathrm{M}^{2} \mathrm{~K} / \mathrm{BT}$ - для второй зоны; $\mathrm{R}_{3}=8,6 \mathrm{M}^{2} \mathrm{~K} / \mathrm{BT}^{2}$ - для третей зоны, и для $\mathrm{R}_{4}=14,2 \mathrm{M}^{2} \mathrm{~K} / \mathrm{BT}$ - для четвертой зоны [1].

Теплопотери через неутепленный пол рассчитываются по формуле

$$
Q=\left(t_{\mathrm{B}}-t_{\mathrm{H}}\right) \sum_{\mathrm{i}=1}^{4} \frac{F_{i}}{R_{i}} .
$$


Очевидно, что такой подход не учитывает форму, а также другие характерные особенности каждого здания, и позволяет лишь приближенно оценить потери теплоты через фундамент. При его использовании не учитывается также реальная теплопроводность грунта и естественное изменение температуры по его глубине. Как известно, ниже некоторой глубины $H$, значение которой зависит от геологических процессов, характерных для данной местности, температурный режим - стабильный в течение всего года [2]. Как показано в [3], для Киева, например, на глубине $H \sim 5$ м температура грунта в течение всего года практически не изменяется и составляет $t_{\text {г }} \sim 8,9 \ldots 9,1^{\circ} \mathrm{C}$.

Для уточнения теплофизической модели переноса теплоты из помещения через неутепленный фундамент в окружающую среду проводится расчетный анализ данного процесса на основе решения уравнения теплопроводности для грунтового массива, на котором расположено здание.

\section{Постановка задачи}

Температурное поле в грунтовом массиве описывается уравнением теплопроводности

$$
\frac{\partial^{2} t}{\partial x^{2}}+\frac{\partial^{2} t}{\partial y^{2}}+\frac{\partial^{2} t}{\partial z^{2}}=0
$$

Область решения уравнения (2) представляет собой прямоугольный параллелепипед, охватывающий некоторый участок грунтового массива. На его поверхности $(\mathrm{z}=0)$ расположено здание прямоугольной формы, Здание занимает участок поверхности: $-x_{\text {зд }} \leq x \leq x_{3 д}$; $-y_{\text {зд }} \leq y \leq y_{\text {зд. }}$ Вследствие предполагаемой симметрии здания (плоскости симметрии: $x=0$; $y=0$ ) задача теплопереноса решается для части области, прилегающей к одной четверти здания. Поверхность $z=0$ расчетной области соответствует поверхности грунта, одна часть которой расположена под прямоугольным зданием $\left(0 \leq x \leq x_{\text {зд }} ; 0 \leq y \leq y_{\text {зд }}\right)$, а другая часть $\left(x_{\text {зд }}<x \leq x_{\max } ; y_{\text {зд }}<y \leq y_{\max }\right)-$ вне здания. Участки $\left(x_{\text {зд }}^{\text {3д }} \Delta_{\text {ст }}<x<x_{\text {зд }} ; y_{\text {зд }}-\Delta_{\text {ст }}<y<y_{\text {зд }}\right)$ соответствуют стенам здания. На поверхности грунта, находящейся под зданием, происходит теплоотдача от воздуха внутри помещения к грунту. Вне здания происходит теплоотдача от поверхности грунта к наружному воздуху. Участки здания, соответствующие боковым стенам, считаются изолированными для тепловых потоков, направленных вдоль оси 0Z. В соответствии с изложенным, на указанных участках поверхности z = 0 для уравнения (2) задаются граничные условия в виде:

- при $z=0 ; 0 \leq x \leq x_{\text {зд }}-\Delta_{\text {ст }} ; 0 \leq y \leq y_{\text {зд }}-\Delta_{\text {ст }}$ :

$-\lambda_{\mathrm{r}} \frac{\partial t}{\partial z}=\alpha_{\mathrm{B}}\left(t-t_{\mathrm{B}}\right)$

- при $z=0 ; x_{\text {зд }}-\Delta_{\text {ст }}<x \leq x_{\text {зд }} ; y_{\text {зд }}-\Delta_{\text {ст }}<y \leq y_{\text {зд }}$ :

$\frac{\partial t}{\partial z}=0$

- при $z=0 ; x_{\text {зд }}<x \leq x_{\text {max }} ; y_{\text {зд }}<y \leq y_{\text {max }}$ :

$-\lambda_{\mathrm{\Gamma}} \frac{\partial t}{\partial z}=\alpha_{\mathrm{H}}\left(t-t_{\mathrm{H}}\right)$.

Температура воздуха вне здания $t_{\mathrm{H}}$ соответствует условиям зимнего периода года. Неутепленный фундамент здания занимает участок расчетной области $-\Delta_{\phi} \leq z \leq 0 ; 0 \leq x \leq x_{\text {зд; }}$; $0 \leq y \leq y_{\text {зд. }}$ Термическое сопротивление фундамента в условиях (3) - (5) не содержится, поскольку его теплопроводность считается близкой к теплопроводности грунта, и он рассматривается как часть грунтового массива.

На горизонтальной поверхности расчетной области $z=-H$ задается температура грунта $t$, которая считается постоянной и не зависящей от температуры наружного воздуха $t_{\mathrm{H}}$ :

- при $z=-H ; 0 \leq x \leq x_{\max } ; 0 \leq y \leq y_{\max }: t=t_{\mathrm{r}}$.

Вертикальные плоскости $x=0 ; y=0$ считаются плоскостями симметрии здания. Вертикальне плоскости $x=x_{\max } ; y=y_{\max }$, ограничивающие расчетную область, считаются теплоизолированными. Таким образом, граничные условия на боковых поверхностях расчетной области записываются в виде:

при $-H \leq z_{\text {зд }} \leq 0 ; x=0 ; x_{\max }$ :

$\frac{\partial t}{\partial y}=0$.

при $-H \leq z_{\text {зд }} \leq 0 ; y=0 ; y_{\max }$ :

$\frac{\partial t}{\partial x}=0$.

Решение уравнения (2) с граничными условиями (3)-(8) выполняется методом конечных разностей. Теплопотери здания через неутепленный 
фундамент рассчитываются по формуле:

$Q=\left.4 \lambda_{\Gamma} \int_{0}^{x_{33}-\Delta_{\text {сг }}} \int_{0}^{y_{\text {зд }-\Delta_{\text {сг }}}} \frac{\partial t}{\partial z}\right|_{z=0} d y d x$

\section{Анализ результатов численного исследования}

В качестве примера рассматривается теплоперенос через неутепленный фундамент здания, основание которого имеет размеры $2 y_{\text {зл }}=60 \mathrm{M}$; $2 x_{\text {зд }}=18 \mathrm{м}$; толщина стен $\Delta_{\text {ст. }}=0,3$ м. Для решения задачи принимаются следующие значения определяющих параметров: $x_{\max }=19 \mathrm{~m} ; y_{\text {зд }}=40 \mathrm{M}$; $H=6 \mathrm{M} ; t_{\mathrm{p}}=20{ }^{\circ} \mathrm{C} ; t_{\mathrm{r}}=8{ }^{\circ} \mathrm{C} ;-15{ }^{\circ} \mathrm{C} \leq t_{\mathrm{H}}{ }^{3 \mathrm{M}}+5{ }^{\circ} \mathrm{C}$; $\alpha_{\mathrm{H}}=23 \mathrm{BT} /\left(\mathrm{M}^{2} \mathrm{~K}\right) ; \alpha_{\mathrm{B}}=8,6 \mathrm{BT} /\left(\mathrm{M}^{2} \mathrm{~K}\right) ; \lambda_{\Gamma}=0,993^{\mathrm{H}} \mathrm{BT} /(\mathrm{M} \cdot \mathrm{K})$. Распределение температуры в плоскости $x=0$ грунтового массива для случая $t_{\mathrm{H}}=0^{\circ} \mathrm{C}$ представлено на рис. $1, a$. Стрелками на рисунке обозначены направления тепловых потоков в данной плоскости. Для сравнения, на рис. 1, б представлены результаты решения аналогичной задачи, полученные при условии, что плоскость $z=-H-$ теплоизолирована. В этом случае при решении задачи вместо условия (6) используется условие

$-\left.\lambda_{\Gamma} \frac{\partial t}{\partial z}\right|_{z=-H}=0$.

Как видно из рис. $1, a$, в случае задания стабильной температуры $t$ г на нижней границе расчетной области $z=-H$, значение которой выше, чем $t_{\text {н }}$, но ниже, чем $t_{\text {в }}$ на указанной границе наблюдаются тепловые потоки, имеющие как положительное так и отрицательное направления. Отрицательные тепловые потоки характерны для области, расположенной под зданием, а положительные - для области вне здания. Из этого следует, что из здания через фундамент теплота переносится не только во внешнее воздушное пространство, но и в глубину грунтового массива. Данное обстоятельство, по-видимому, не учитывается выражением (1), которое обычно используется при расчете тепловых потерь через фундамент. В случае же задания условий теплоизоляции при $z=-H$, теплота из здания переносится только к наружному воздуху (рис. 1, б).

Распределение плотности теплового потока на поверхности грунтового массива $(z=0)$ вдоль оси $0 \mathrm{X}$ при $y=0$ (плоскость симметрии) и при $y$ $=y_{\text {зд }}-\Delta_{\text {ст }}$ (около стены) представлено на рис. 2 . Рассматриваются случаи температуры во внеш- нем воздушном пространстве $t_{\mathrm{H}}=+5^{\circ} \mathrm{C}$ и $t_{\mathrm{H}}=-15^{\circ} \mathrm{C}$ при условия (6) на границе $z=-H$. Как видно из рис. 2, плотности тепловых потоков на поверхности неутепленного фундамента, а также на поверхности грунта вне здания интенсивнее всего изменяются на расстоянии $1,5 \ldots 2$ м от стены. Около самих стен они достигают максимальных по абсолютной величине значений. Далее от стен абсолютные значения $q$ уменьшаются. Плотности тепловых потоков, рассчитанные на плоскости симметрии здания $y=0$, при $x<6$ м слабо зависят от значения температуры наружного воздуха (рис. $2, a)$ и составляют $q \sim 2,0 \ldots 2,6 \mathrm{BT} /\left(\mathrm{M}^{2} \mathrm{~K}\right)$ при $t_{\mathrm{H}}=+5{ }^{\circ} \mathrm{C}$ и $q \sim 2,0 \ldots 3,6 \mathrm{BT} /\left(\mathrm{M}^{2} \mathrm{~K}\right)$ при $t_{\mathrm{H}}=-15{ }^{\circ} \mathrm{C}$. Данные величины плотностей тепловых потоков определяются, в основном, разностью температур $t_{\text {в }}$ и $t_{\text {г }}$ Отметим, что значение $q$, рассчитанное по

формуле $\quad q=\frac{t_{\mathrm{B}}-t_{\mathrm{r}}}{1 / \alpha_{\mathrm{B}}+H / \lambda_{\mathrm{r}}}, \quad$ учитывающей только теплоперенос вглубь грунта, составляет $q=1,95 \mathrm{BT} /\left(\mathrm{M}^{2} \mathrm{~K}\right)$. Около стен плотности тепловых потоков зависят в большей степени от разности температур $t_{\mathrm{B}}$ и $t_{\mathrm{H}}$. При $t_{\mathrm{H}}=+5{ }^{\circ} \mathrm{C}$ плотность теплового потока около стены равна $q=23,5 \mathrm{BT} /\left(\mathrm{M}^{2} \mathrm{~K}\right)$, а при $t_{\mathrm{H}}=-15^{\circ} \mathrm{C}-$ соответственно $q=51,4 \mathrm{BT} /\left(\mathrm{M}^{2} \mathrm{~K}\right)$.

Распределение плотностей тепловых потоков вдоль стены при $y=y_{\text {зд }}-\Delta_{\text {ст }}$ представлено на рис. 2, б. Как видно из рисунка, значения $q$ около стены существенно выше, чем на плоскости симметрии здания $y=0$. Эти значения определяются, главным образом, разностью температур $t_{\text {в }}$ и $t_{\text {н }}$. Максимальних по абсолютной величине значений $q$ достигают в углах здания $\left(y=y_{\text {зд }}-\Delta_{\text {ст }} ; x=x_{\text {зд }}-\Delta_{\text {ст }}\right)$. При $t_{\text {н }}=+5{ }^{\circ} \mathrm{C}$ значение $q$ около угла составляет $-41,7 \mathrm{BT} /\left(\mathrm{M}^{2} \mathrm{~K}\right)$, а при $t_{\mathrm{H}}=-15{ }^{\circ} \mathrm{C}-q=-93,4 \mathrm{BT} /\left(\mathrm{M}^{2} \mathrm{~K}\right)$. То обстоятельство, что около углов здания теплопотери максимальны, учитывается также методикой разделения пола зоны. При расчете площади первой зоны участок площадью $2 \times 2$, примыкающий к углу, учитывается дважды [1].

В соответствии с характером изменения теплового потока по неутепленному полу изменяется также и его температура. Минимальные значения температуры наблюдаются около стен, 
а максимальные - в средней части пола. Полученное в результате расчета распределение температуры по полу неутепленного первого этажа рассматриваемого здания представлено на рис. 3. На этом же рисунке представлены также результаты измерения температуры пола, которые проводились в Выставочном зале Института технической теплофизики НАН Украины 4 марта 2015 г. Измерение температуры на поверхности пола производились с помощью тепловизора TESTO 875. Для расчетов принимались экспериментально найденные значения температуры воз-
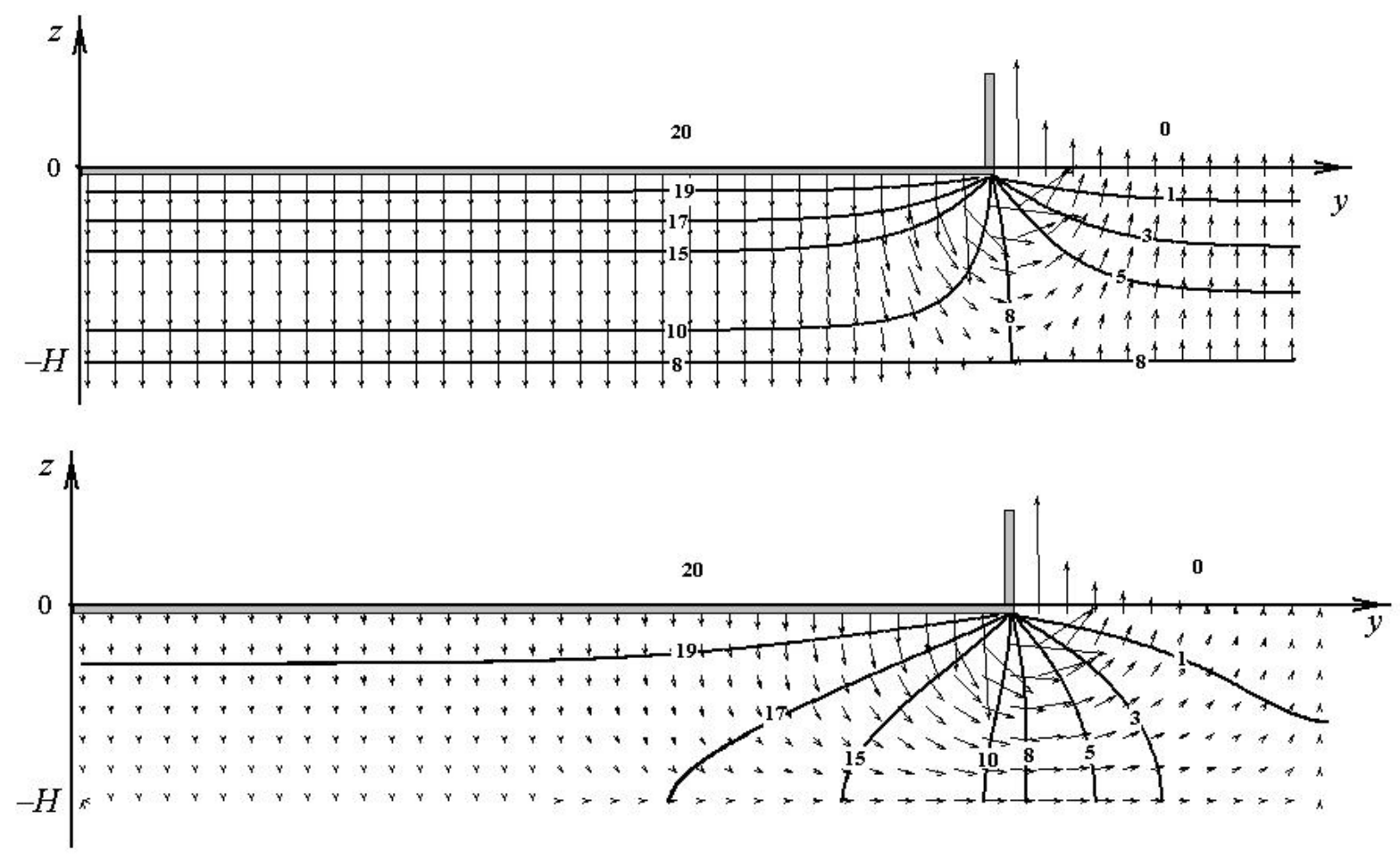

\section{Рис. 1. Распределение температуры и направления тепловых потоков в сечении $x=0$ грунтового массива при $t_{\mu}=0^{\circ} \mathrm{C}$.}

душной среды внутри и снаружи помещения: $t_{\mathrm{H}}=$ $+2{ }^{\circ} \mathrm{C} ; t_{\text {в }}=+13,5^{\circ} \mathrm{C}$ (выставочный зал - слабо обогреваемый). Остальные исходные параметры для решения задачи принимались такими же, как и в рассмотренном выше случае. Как видно из рис. 3, расчетные и экспериментальные результаты удовлетворительно согласуются между собой.

Суммарные теплопотери через фундамент здания целесообразно оценивать по величине удельного теплового потока $U=Q /\left(t_{\text {в }} t_{\mathrm{H}}\right)$, для которого значение $Q$ рассчитывается по формуле (9). Зависимость указанной величины от температуры наружного воздуха tн при постоянной $t_{\text {в }}$ $=20^{\circ} \mathrm{C}$ представлена на рис. 4 (кривая $\left.1, a\right)$. Как видно из рисунка, с уменьшением $t_{\text {н }}$, величина $U$ убывает. Это связано с уменьшением вклада в суммарные теплопотери переноса теплоты в глубину грунта. При понижении $t_{\mathrm{H}}$, т.е. при росте температурного напора $\Delta t=t_{\mathrm{B}}-t_{\mathrm{H}}$, теплота из здания через фундамент переносится, в основном, к наружному воздуху, поскольку в этом случае $t_{\text {н }}$ значительно ниже, чем $t_{\text {г }}$ Вклад переноса теплоты вглубь грунтового массива в суммарные теплопотери оказывается при этом незначительным. При понижении температуры $t$ значения $U$ оказываются близкими к величине $U^{*}$, которая находится из решения данной задачи при условии (10), т.е. при условии теплоизоляции поверхности $z=-H$ (линия 2, $a$ ).

На рис. 4 приводится также значение удельного теплового потока $U$, найденного из выражения (1), которое используется при расчете теплопо- 
терь зональным методом (кривая 3, a). Сравнивая значения удельных тепловых потоков, найденных различными способами, модно отметить, что в рассматриваемом диапазоне температуры наружного воздуха $t_{\text {н }}$ величины $U$, полученные из решения задачи з граничным условием (6), занимают промежуточное значение между величина-

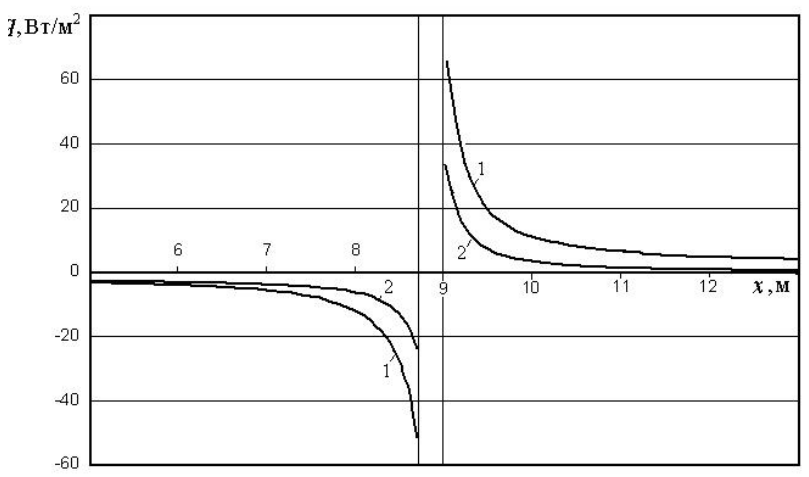

a) ми $U^{*}$, найденными из решения задачи с условиями теплоизоляции при $z=-H$, и величинами $U$, полученными из выражения (1). Следует, однако, отметить, что рассмотренный выше зональный метод, а также соответствующие ему значения термических сопротивлений каждой зоны, рекомендованы для фундаментов и грунта, тепло-

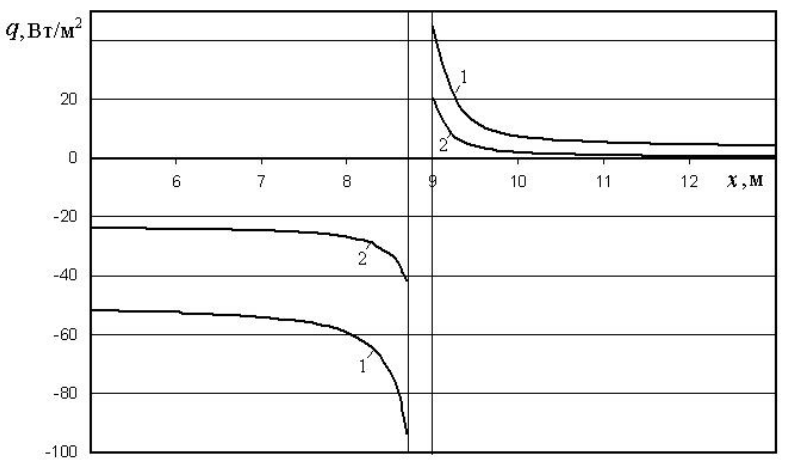

б)

Рис. 2. Распределение плотности теплового потока по поверхности пола вдоль оси ОХ при у $=0$ (а) и при $y=y_{\text {зо }}-\Delta_{c m}$ (б): $1-t_{u}=-15^{\circ} \mathrm{C} ; 2-t_{u}=+5^{\circ} \mathrm{C}$.

проводность которых превышает 1,2 Вт/(м·К). Результаты расчета тепловых потерь, найденные методом численного моделирования, получены при $\lambda_{г}=0,993 \mathrm{BT} /(\mathrm{M} \cdot \mathrm{K})$.

Рассмотренные выше результаты относятся к зданию, фундамент которого имеет форму прямоугольника, длина которого более чем в три раза превышает его ширину. Для выяснения влияния геометрической формы фундамента на значение удельных потерь теплоты через указанный фундамент, рассмотрим результаты их расчета для здания с фундаментом квадратной формы. Площадь квадратного фундамента при этом равна площади прямоугольного фундамента, рассмотренного выше. Указанные результаты представлены на рис. 4 кривыми, имеющими индекс (б). Как видно из рисунка, удельные тепловые потоки через фундамент квадратного сечения оказываются ниже аналогичных величин, полученных для фундамента прямоугольного сечения той же площади. При этом значения $U^{*}$, найденные из решения задачи с условиями теплоизоляции при $z=-H$, отличаются в 1,2 раза, величины $U$, полученные с использованием формулы (1), - в 1,14 раза, а $U$, рассчитанные с граничным условием (6), - в $1,09 \ldots 1,12$ раза.

\section{Выводы}

1. Из представленных результатов численного исследования тепловых потерь через неутепленный фундамент следует, что теплоперенос вглубь грунта оказывает заметное влияние на суммарные теплопотери в случае близких по значению температуры наружного воздуха $t_{\text {н }}$ ием-

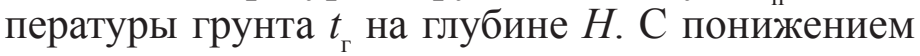
температуры наружного воздуха указанное влияние ослабевает.

2. Плотности тепловых потоков на поверхности неутепленного пола увеличиваются по абсолютной величине в направлении стен здания. Максимальные тепловые потери через пол наблюдаются в области углов здания. Температура неутепленного пола по направлению к стенам уменьшается.

3. Тепловые потери через фундамент прямоугольной формы, у которого длина и ширина заметно отличаются, выше, чем через квадратный фундамент такой же площади. 


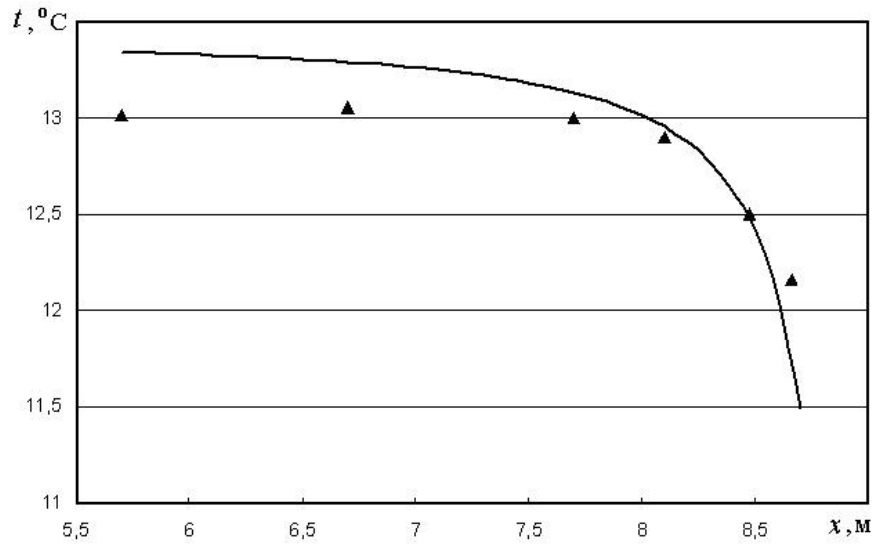

Рис. 3. Распределение температуры по поверхности пола вдоль оси ОХ при у $=0$ (плоскость симметрии): $t_{n}=+2^{\circ} \mathrm{C} ; t_{b}=+13,5^{\circ} \mathrm{C}$; сплошная линия-расчет; треугольники - эксперимент.

\section{ЛИТЕРАТУРА}

1. Богословский В.Н., Сканави А.Н. Отопление.М: Стройиздат. - 1991. - 735 с.

2. Накорчевский А.И., Беляева Т.Г. Регрессионный анализ глубин годовых флуктуаций темпе-

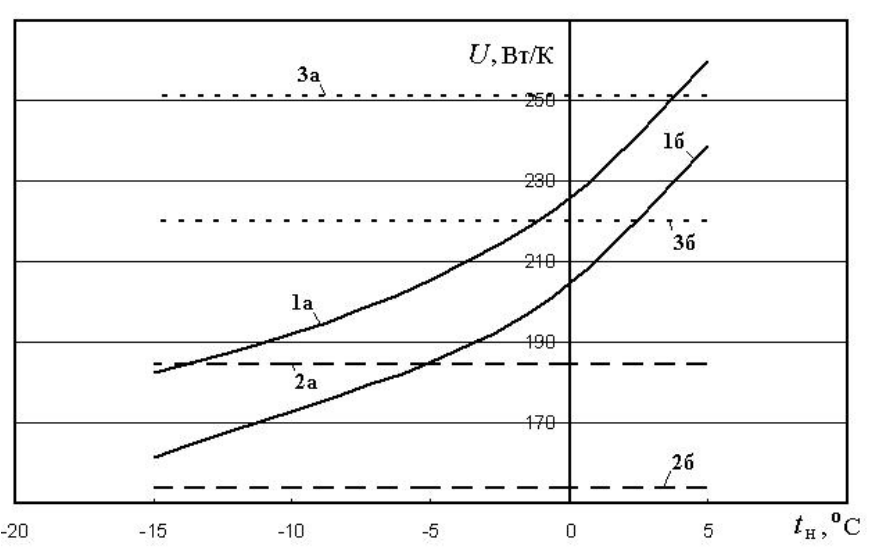

Рис. 4. Зависимость удельных потерь теплоты через неутепленный фундамент от температуры наружного воздуха: а-здание с прямоугольным фундаментом; б - здание с квадратным фундаментом.

ратур в верхних слоях грунта // Промышленная теплотехника. - 2005. - т.27, № 6. - С. 86 - 90.

3. Накорчевский А.И. Грунтовые аккумуляторы теплоты и модернизация коммунальной теплоэнергетики. Киев «Наукова думка». -2010. - 256 с. 


\section{NUMERICAL SIMULATION OF HEAT TRANSFER THROUGH NON-INSULATED BUILDING FOUNDATION}

\section{Basok B.I., Davydenko B.V., Novikov V.G., Goncharuk S.M., Lysenko O.M.}

Institute of Engineering Thermophysics of the National Academy of Sciences of Ukraine, vul. Zhelyabova, 2a, Kyiv-57, 03057, Ukraine

The heat transfer from the room through the noninsulated foundation to environment is investigated by numerical modeling. Numerical analysis of this process is carried out taking into account the temperature stabilization of ground array at a certain depth. Dependence of the relative heat flux through the foundation of the building on the outdoor temperature is obtained. It is shown that the losses of heat through the foundation of square shape are smaller than through the foundation of rectangular shape whose length is considerably greater than the width. The result of calculating of the temperature distribution on the surface of the non-insulated floor is in a good agreement with the experimental data.

References 3, figures 4 .

Key words: foundation of the building, heat loss, numerical modelin.

1. Bogoslovskiy V.N., Skanavi A.N. Heating - M: Stroyizdat. - 1991. -735 p.

2. Nakorchevskiy A.I., Belyaeva T.G. Regression analysis of the depth of annual fluctuations in temperature in the upper layers of soil // Promyishlennaya teplotehnika. - 2005. - v.27, No 6. - P. $86-90$.

3. Nakorchevskiy A.I. Ground heat batteries and modernization of municipal power system. - Kiev: «Naukova dumka». -2010. - 256 p.

Получено 30.03.2015 Received 30.03.2015 